\title{
CORR Rachel, Ritual and remembrance in the Ecuadorian Andes
}

\author{
Juan Javier Rivera Andía
}

Traducteur : Philippe Erikson

\section{OpenEdition \\ Journals}

Édition électronique

URL : https://journals.openedition.org/jsa/12431

DOI : 10.4000/jsa.12431

ISSN : $1957-7842$

\section{Éditeur}

Société des américanistes

Édition imprimée

Date de publication : 31 décembre 2012

Pagination : 248-255

ISSN : 0037-9174

\section{Référence électronique}

Juan Javier Rivera Andía, "conr Rachel, Ritual and remembrance in the Ecuadorian Andes ", Journal de la Société des américanistes [En ligne], 98-2 | 2012, mis en ligne le 05 juin 2013, consulté le 04 septembre 2022. URL : http://journals.openedition.org/jsa/12431; DOl : https://doi.org/10.4000/jsa. 12431

Ce document a été généré automatiquement le 4 septembre 2022.

Tous droits réservés 


\title{
CORR Rachel, Ritual and remembrance in the Ecuadorian Andes
}

\author{
Juan Javier Rivera Andía \\ Traduction : Philippe Erikson
}

\section{RÉFÉRENCE}

CORR Rachel, Ritual and remembrance in the Ecuadorian Andes, University of Arizona Press, Tuscon, 2010, xi + $184 \mathrm{p}$.

1 Ce travail vient enrichir l'œuvre que Rachel Corr consacre, depuis près de dix-huit ans, aux représentations collectives des habitants du village de Salasaca, situé dans la province équatorienne de Tunghurahua à environ 2700 mètres d'altitude. L'auteur lui avait déjà dédié une thèse de doctorat soutenue voilà plus de dix ans à l'université d'Illinois (Corr 2000).

Nous commencerons par une discussion du contenu du livre, avant de nous intéresser de plus près à quelques-uns des concepts et préceptes méthodologiques qui y sont utilisés. Bien que l'ouvrage ne présente aucune réflexion de fond sur ce point, la première chose qui saute aux yeux est que Salasaca est loin d'être une enclave coupée du reste du pays: ce village de 12000 habitants bilingues (hispano- et quechua-phones) n'est situé qu'à trois heures et demie de bus de l'aéroport international de Quito et compte aujourd'hui bon nombre de constructions en béton et tôle ondulée (p. 83). Rien d'étonnant, dès lors, à ce que les Salasaqueños utilisent la métaphore des "rayons $\mathrm{X}$ » pour décrire leurs pratiques curatives traditionnelles; ou encore qu'ils expliquent leurs croyances en la multiplicité des âmes ${ }^{1}$ en les comparant à des « photographies » (p. 121). Leurs échanges ne se cantonnent d'ailleurs pas au niveau national équatorien, puisqu'ils impliquent également des touristes venus du monde entier, attirés par les fêtes qui se déroulent dans leur village (p. 63). Notons, en outre, qu'aujourd'hui ce sont souvent des émigrés revenus d'Espagne qui occupent les charges rituelles (mayordomías), ce qui, on s'en doute, a eu tendance à favoriser la dimension spectaculaire de ces fêtes au détriment de leur 
religiosité. À l'évidence, Salasaca illustre parfaitement le cas, souvent décrit au Pérou depuis les années 1970, de ces villages "tournés vers l'extérieur", dont des familles entières ont migré vers la ville, laissant leurs maisons et leurs champs semi-abandonnés.

Cette omniprésence de l'Équateur moderne ressortait déjà d'une publication antérieure de l'auteur, consacrée à l'auto-exotisation de Salasaca par les habitants eux-mêmes lors de l'élection des protagonistes rituels (Corr 2003). Elle concorde en outre par divers aspects avec l'image "politisée " du monde indigène équatorien que nous ont récemment livrée certains ethnologues (Moreno 2008). On trouve, par exemple, dans le chapitre VI du livre dont il est ici question, une description de chamanes ${ }^{2}$ organisés en associations provinciales, avec des programmes de formation qui délivrent des " certificats» (p.116), habitués à facturer leurs services (p.115) et apparentés avec les candidats présidentiels (p. 107) ${ }^{3}$.

Corr nous apprend, dans le même ordre d'idées, que les Salasaqueños se considèrent membres de la grande « fratrie indigène » de l'Équateur (p. 114), au sein de laquelle ils constitueraient un groupe ethnique distinct ${ }^{4}$. Elle ajoute qu'ils se disent fiers de préserver de nombreux aspects des rituels de leurs ancêtres (p.16). Cependant, aucune précision n'est apportée pour expliquer comment de telles affirmations s'insèrent dans le contexte politico-régional.

5 Cette lacune est surtout frappante lorsque Corr prête, à ce propos, une sorte de volonté collective à ses hôtes, affirmant par exemple : «Salasacans selected and manipulated aspects of Catholic discourse and ritual» (p. 16). Une telle propension se retrouverait même chez ceux que l'on pourrait supposer les plus indifférents aux «traditions» ou à la « religiosité » indigènes, tels les protestants «qui s'efforcent cependant de maintenir autant que possible les traditions culturelles sans trahir leurs croyances religieuses $»^{5}$. Selon l'auteur, pareille volonté de manipuler le discours et le rituel catholiques présenterait, en outre, une certaine stabilité dans le temps. Ainsi, au début du xx siècle, les Salasaqueños auraient-ils tenté d'influer sur certains aspects de l'évangélisation en cours $^{6}$ et de contrôler leurs expériences religieuses, voire la nature même de leurs relations avec l'Église (p. 29). Une phrase comme celle qui suit résume parfaitement le fil conducteur de son argumentation: «dans le contexte de l'Équateur indigène postcolonial, les gens s'efforçaient de garder le contrôle de leur vie religieuse ${ }^{7}$.

Corr utilise de telles hypothèses pour expliquer, par exemple, pourquoi les Salasaqueños ont tant insisté pour que leur village récupère telle image catholique ou pourquoi ils ont renoncé à couper un arbre de quishuar (Buddelei longifolia) : certaines lettres de 1907 laissent en effet entendre qu'ils le révéraient, pensant même qu'il accoucherait sous peu d'une statue de Saint Buenaventura (p. 27). Cette grille lui sert aussi pour interpréter le mythe d'origine d'un rituel au cours duquel un groupe d'enfants fonde une fête de la Vierge à la suite d'un jeu consistant à célébrer son image sur un arbre. Ainsi, selon l'auteur, le système des charges serait la quintessence de la domination coloniale transformée en symbole de l'identité indigène (p. 40). Les indigènes de Salasaca auraient transformé les rôles de leurs mayordomos de manière à ce qu'ils préservent la mémoire culturelle indigène (p.52). Rien ne vient toutefois étayer cette affirmation, aucune description détaillée de l'ancien système des charges ou de l'ancien rôle des mayordomos n'étant donnée ${ }^{8}$. En fait, après avoir examiné tous les exemples fournis, on pourrait même se demander s'il ne serait pas plus pertinent de réinterpréter ces aspects de la vie religieuse de Salasaca en faisant l'économie de cette hypothétique volonté collective de transmission d'une mémoire propre. 
7 Une telle emphase sur l'auto-détermination et le pouvoir de négociation indigènes repose en effet sur la certitude qu'il existerait une "religion dominante », en dépit de laquelle les Salasaqueños auraient continué leurs propres pratiques religieuses ${ }^{9}$. Nous voici donc confrontés une fois de plus au cadre interprétatif bien connu fondé sur deux concepts-clés : domination et résistance indigène (p. 163). De fait, Corr ne laisse aucune place au doute sur ce point lorsqu'elle affirme que «les populations indigènes ont été forcées à se convertir au catholicisme » (ibid.).

8 L'auteur affirme avoir systématiquement choisi les thèmes de son ouvrage en s'efforçant d'éviter deux clichés courants concernant les Amérindiens : celui de victimes, dont la culture aurait été détruite par le colonialisme, d'une part, et celui de peuples qui résistent en occultant leur culture authentique, d'autre part. Il semble évident que les cultures indigènes n'ont été ni annihilées ni contraintes à se camoufler. Corr souhaite, avec cette double négation, insister sur le pouvoir de négociation d'un peuple indigène comme celui de Salasaca. On peut toutefois relever une certaine incongruité à nier que les cultures aient été détruites, tout en continuant à défendre la thèse de la domination écrasante. Si l'on retient l'idée que les stratégies de résistance culturelle indigènes reposaient sur la négociation, voire sur la manipulation, pourquoi, dès lors, retenir également le schème de l'écrasante domination religieuse?

De fait, les aspects des rites de Salasaca ${ }^{10}$ qui retiennent le plus l'intérêt de Corr s'expliquent surtout par sa volonté d'éviter ces deux écueils interprétatifs : la recréation permanente de la religion de par sa pratique individuelle, qui conduit l'auteur à mettre l'accent sur la diversité des actes selon les individus (p.14) ; la réinterprétation du dogme catholique en vue de la préservation de la mémoire culturelle indigène ${ }^{11}$.

Pour illustrer les recréations individuelles de la religiosité des Salasaqueños, Corr présente quelques récits d'expériences de mort imminente, pensant y déceler la forte empreinte de l'évangélisation coloniale sur les consciences individuelles (p.143). On aurait là, pense l'auteur, des réélaborations indigènes de l'imaginaire catholique romain (p. 144). La lecture de ces témoignages ${ }^{12}$ de gens qui affirment être revenus de la mort incite cependant à se demander s'ils n'auraient pas plutôt été influencés par les images véhiculées de nos jours par le cinéma, la télévision et la radio, surtout en des endroits aussi « branchés » que Salasaca.

11 Dans le cadre de son analyse de la réinterprétation du catholicisme et de la mémoire indigène, Corr s'intéresse aux circonvolutions rituelles des Salasaqueños suivant les «chemins sacrés» du village. Ces trajets seraient l'expression de leur identité et ranimeraient ce qu'elle appelle leur « mémoire non verbale ». Les rituels qui, tels Corpus Christi, transitent par les chemins sacrés du village auraient donc pour but - plus ou moins évident dans une optique fonctionnaliste - de manifester « une identité partagée et une tradition commune $»^{13}$. Corr fournit d'autres exemples sur les moyens utilisés par les habitants de Salasaca pour préserver leur mémoire culturelle : l'élaboration annuelle de listes de proches défunts en vue « d'honorer les morts » (p. 98) ; les prières catholiques que les adultes apprennent par cœur en espagnol pour bénir la nourriture et se souvenir de leurs ascendants. Ici encore, pour l'auteur, ces deux pratiques - que nous avons également observées dans la sierra de Lambayeque, au nord du Pérou - serviraient à exprimer une identité commune (p. 73) et à promouvoir une "mémoire généalogique indigène » (p. 82). Corr signale que cette référence aux ascendants défunts dans certaines des prières apprises par cœur aboutit à les réinsérer dans la rhétorique quechua. Mais qu'entendre au juste par "rhétorique quechua» ou «discours culturel andin», que 
l'auteur ne caractérise qu'en évoquant trois traits principaux - l'emphase sur les ancêtres (p.74), l'évocation de femmes et d'hommes (p.72) et la volonté de transmettre une certaine sagesse aux générations suivantes (ibid.) ?

Ayant passé en revue les principales thématiques abordées dans ce livre, examinons à présent certains des fondements méthodologiques, à commencer par une réflexion sur la place à accorder aux interprétations des autres. Corr affirme :

Je ne suis pas favorable aux analyses anthropologiques surajoutées aux explications données par les indigènes, de même que l'analyse interprétative n'occulte pas ces dernières [...]. J'ai souvent discuté de mes interprétations anthropologiques du symbolisme avec les Salasacan [...]. J'essaie de suggérer des interprétations sans les plaquer sur les représentations indigènes de leurs propres pratiques. ${ }^{14}$

Voilà qui laisse entendre une certaine tension entre l'interprétation anthropologique des faits et la vision indigène des choses. Mais pourquoi les explications des ethnologues excluraient-elles celles des habitants de Salasaca qui s'imposent à leur détriment? $\mathrm{Ne}$ s'alimentent-elles pas plutôt les unes les autres au cours d'un jeu de réajustements constants selon les différents interlocuteurs (politiciens, intellectuels, gens des médias, militants, indigénistes, etc.) ? On voit mal quel est ce malaise qui incite Corr à prendre tant de précautions qui semblent en fin de compte contre-productives.

Examinons, par exemple, son interprétation du jeu rituel appelé "huayru » qui se pratique après le décès d'un parent. Bien qu'il s'agisse à l'évidence de la partie la plus dense de son ouvrage au niveau ethnographique, Corr se contente de répéter l'explication des Salasaqueños, à savoir que ce jeu servirait à " rendre l'âme heureuse ». Un constat similaire s'impose à propos de la description des caporales, version locale de la danza de negros si répandue au sein des populations indigènes des Andes péruviennes et boliviennes. L'auteur se contente d'affirmer, répétant ce qui semble se dire dans le village, qu'il s'agirait d'une danse représentant « des soldats afro-équatoriens de la côte » (p. 74) qui auraient été vus, en 1895, lors de la révolution libérale de l'Équateur. Corr affirme vouloir se prémunir contre la surimposition d'une interprétation sur une autre, mais cela revient, en fin de compte, à proposer aux ethnologues de se cantonner dans la simple paraphrase, sans faire plus que reprendre platement les propos indigènes. Pourquoi ne pas tenir compte des deux types d'interprétations, sans les hiérarchiser, ni les substituer l'une à l'autre ? Citons par exemple ce que fait Erikson (2011, p. 112) :

Pour les Matis, la signification de ceci est claire : le mariwin étant une sorte de surhomme, il est normal qu'il ait des ornements extraordinaires [...]. L'ethnologue, pour sa part, en tirera des conclusions plus mélancoliques, [... lisant] dans la comparaison des masques et des visages contemporaines, le déclin d'une civilisation.

La seconde question de méthode que nous souhaitons signaler est celle de la place accordée au comparatisme dans l'analyse des pratiques culturelles des Salasaqueños. Corr mentionne certes les travaux produits par Foster $(1960)^{15}$. Cependant, à l'instar de la majorité de nos collègues andinistes, elle ne se réfère pratiquement jamais aux parallélismes possibles avec des faits provenant d'Espagne ou d'autres parties du monde hispanique. Considérons son interprétation du culte à un "arbre sacré " (pp. 30-31). L'auteur suggère, de manière assez classique, qu'il s'agirait là de la continuation d'une tradition andine précolombienne (p. 34). Elle ne livre guère plus d'informations sur cette filiation et ne dit rien des cas similaires situés hors de l'aire andine équatorienne. La ressemblance est pourtant frappante entre ce qui est décrit pour Salasaca et ce que l'on trouve dans les écrits de Martínez Compañón (1936), évêque de Trujillo (au nord du 
Pérou) à la fin du XVIII ${ }^{e}$ siècle. Aujourd'hui encore, lors de notre travail de terrain dans une partie de cette vaste région, nous avons observé que des offrandes étaient faites à des arbres, avec lesquels les gens communiquaient par rêve et qu'ils associaient à des images catholiques. On pourrait toutefois aller plus loin encore que la simple comparaison synchronique limitées aux frontières nationales andines ${ }^{16}$.

Dans le même ordre d'idées, mais à une plus vaste échelle, signalons qu'il aurait été utile d'éclairer les mythes de Salasaca sur l'apparition des Saints à la lumière du fameux système de classification des motifs ATU (Aarne-Thompson-Uther), comme le signalait déjà Efrain Morote Best dans les années 1950. Peut-être vaut-il la peine d'ajouter un dernier exemple: le nettoyage rituel de la maison du défunt (appelé ucu pichana) qui s'effectue après l'enterrement. L'auteur nous indique qu'on balaie alors la maison avec deux éléments qui apparaissent également lorsqu'il s'agit de "nettoyer» le corps d'un malade (p. 95) : un cochon d'Inde et certaines " plantes médicinales ${ }^{17}$. Hormis cette référence contemporaine à Salasaca, Corr puise tout le reste de ses références interprétatives dans le monde andin précolombien ${ }^{18}$. Pourquoi se priver ainsi des sources historiques et ethnographiques portant sur l'Europe et plus particulièrement sur la péninsule ibérique? Un ouvrage consacré à un univers aussi métissé et hispanique que Salasaca aurait dû accorder une plus large place au comparatisme, suivant une démarche dont la pertinence est régulièrement signalée depuis plus d'une dizaine d'années :

On ne saurait trop recommander aux futurs américanistes la pratique de l'ethnographie espagnole sous forme de lectures, mais aussi de "terrain »: on recherche généralement les influences ibériques dans les grandes institutions, dans une christianisation de doctrine, mais rarement dans la réalité des pratiques et la profondeur des croyances, dans les réélaborations inconscientes de thèmes missionnaires, dans l'invention de rites et de croyances. Pour relever ces combinaisons complexes, il est indispensable de prendre en considération l'ethnographie espagnole dans toute sa diversité contemporaine. En effet, ces influences ont été complétement occultées jusqu'à nos jours pour des raisons idéologiques. (Molinié 2001, p. 224)

Une dernière remarque, avant de conclure, sur la récurrence de l'adjectif « sacré » tout au long de l'ouvrage que nous venons de recenser et que Rachel Corr utilise pour qualifier divers paysages ${ }^{19}$, lieux, chemins et actes de Salasaca (p. 13). Bien qu'omniprésent, le terme n'est jamais défini, ni discuté, comme si son emploi allait de soi...

En fin de compte, cet ouvrage est susceptible d'intéresser les spécialistes des récits et rituels contemporains de l'aire andine. Certes, tant l'approche de l'auteur que les méthodes et concepts qu'elle déploie mériteraient d'être affinés. L'ouvrage n'en propose pas moins un matériau ethnographique riche et les nombreuses données qu'il contient pourraient, par le biais d'une comparaison systématique, permettre de lever certains des mystères qui planent encore au-dessus du thème de la relation entre rite et mémoire collective dans les Andes. 


\section{BIBLIOGRAPHIE}

CORR Rachel

2000 Cosmology and personal experience : representations of the sacred landscape in Salasaca, Ecuador, PhD., University of Illinois, Urbana-Champaign.

2003 « Rituals, knowledge and the politics of identity in Andean festivities », Ethnology, 42 (1), pp. 39-54.

\section{DIEZ Alejandro}

2005 « Los sistemas de cargos religiosos y sus transformaciones ", in Manuel Marzal (éd.),

Religiones andinas, Trotta, Madrid, pp. 253-286.

ERIKSON Philippe

2011 «"Bats-moi, mais tout doucement...". Mascarades cinglantes et pédagogie rituelle chez les Matis (Amazonas, Brésil) », in Jean-Pierre Goulard et Dimitri Karadimas (éds), Masques des hommes, visages des dieux. Regards d'Amazonie, CNRS, Paris, pp. 107-127.

FOSTER George M.

1960 Culture and conquest : America's spanish heritage, Wenner-Gren Foundation, coll. « Viking Fund Publications in Anthropology » 27, New York.

MARTÍNEZ COMPAÑóN Baltazar Jaime

1936 Trujillo del Perú a fines del siglo XVIII: dibujos y acuarelas que mandó hacer el Obispo D. Baltazar Jaime Martínez Compañón, Cultura Hispánica del Centro Iberoamericano de Cooperación, Madrid. MOLINIÉ Antoinette

2001 «Les études américanistes. Andes », in Martine Segalen (éd.), Ethnologie. Concepts et aires culturelles, Armand Colin, Paris, pp. 218-228.

\section{MORENO Segundo}

2008 « Antropología abierta, antropología ambigua: tendencias actuales de la antropología ecuatoriana ", in Alejandro Diez (éd.), La antropología ante el Perú de hoy. Balances regionales y antropologías latinoamericanas, PUCP-CISEPA, Lima, pp. 205-218.

\section{PÉREZ GALÁN Beatriz}

2004 Somos como incas : autoridades tradicionales en los Andes peruanos, Cuzco, Iberoamericana/ Vervuert, Madrid/Frankfort.

ROBIN Valérie

2008 Miroirs de l'autre vie. Pratiques rituelles et discours sur les morts dans les Andes de Cuzco (Pérou), Société d'ethnologie, coll. « Recherches américaines » 7, Nanterre.

\section{NOTES}

1. Ce point aurait gagné à être revu à la lumière des ethnographies récentes consacrées à cette thématique dans les Andes péruviennes (voir Robin 2008).

2. L'ouvrage ne fournit pas les noms donnés à ces spécialistes par les Salasaqueños.

3. Le chapitre dévolu au traitement des maladies par le biais d'offrandes aux sommets aurait pu faire meilleur usage des innombrables études consacrées aux croyances et pratiques entourant les montagnes dans les Andes péruviennes. On ne trouve pas, non plus, de dialogue avec les 
travaux consacrés au chamanisme tel qu'il se pratique dans les provinces péruviennes limitrophes ou dans les sociétés du piémont andin septentrional.

4. "People are aware of the uniqueness of their customs" (p. 162).

5. « Do however strive to maintain as many cultural traditions as possible without violating their religious beliefs» (p. 16).

6. «[Salasacans] attempted to control some aspects of their ongoing evangelization » (p. 25).

7. "In the context of postcolonial Ecuador, indigenous individuals fought for control over their own religious lives » (p. 53).

8. À Salasaca, comme dans une bonne partie des Andes du Nord du Pérou, les charges rituelles (mayordomías) se transmettent de manière héréditaire, des parents aux enfants. La situation est différente dans d'autres régions où ce sont plusieurs familles du village qui assument les charges chaque année. Corr ne commente pas les effets de cette différence, pas plus qu'elle ne cite les textes traitant du système des charges dans les Andes (par exemple Diez 2005).

9. «Indigenous people were determined to continue their own religious practices » (p. 38).

10. Corr mentionne trois des rites de Salasaca (p.54): le nouvel an, le Carnaval et Corpus Christi. Ce dernier n'a été décrit qu'à travers les témoignages d'autrui, l'observation directe ayant été interrompue en raison de l'« inconfort physique » du travail de terrain (p. 63). Les cérémonies associées aux défunts, pour une raison ou une autre, n'ont pas été insérées dans cette catégorie.

11. "[Salasacans] recentered catholic rituals to serve as mediums for sustaining indigenous cultural memory... [and] are active in shaping their own religious history » (p. 163).

12. L'auteur nous livre les transcriptions des témoignages en anglais, plutôt qu'en version originale.

13. "A shared identity and a common heritage" (p. 162). Ces réflexions de Corr sur le marquage rituel de l'espace environnant auraient pu être comparées à celles portant sur le même thème dans les Andes péruviennes, notamment celles de Pérez Galán (2004) sur Cuzco.

14. "I do not favor anthropological analysis over indigenous explanations, and I do not view interpretive analysis as precluding indigenous explanations [...]. I often discussed my anthropological interpretations of symbolism with Salasacans [...]. I try to suggest interpretations without imposing on indigenous views of their own practices» (pp. 15-16).

15. On relèvera cependant l'absence d'autres auteurs qui, à la même époque, invoquaient la nécessité de recourir au comparatisme pour comprendre le monde andin.

16. En fait, la comparaison diachronique n'est pas non plus très présente dans ce livre. Connaissant Salasaca depuis plus de 18 ans, l'auteur aurait pourtant pu nous livrer une description détaillée des changements culturels. Corr nous dit que, depuis une dizaine d'années, certains prédisent la disparition des rituels, tandis que d'autres s'efforcent de les préserver (p. 163). On aimerait en savoir plus...

17. L'auteur ne fournit jamais, ni les noms vernaculaires, ni les noms scientifiques des plantes en question.

18. Relevons, en outre, que la plupart des sources utilisées dans ce livre se limitent à la bibliographie andiniste publiée en anglais. Il s'agit certes là d'un corpus important, mais qui omet la plupart des sources parues en Europe et dans les pays andins eux-mêmes.

19. "[Salasacans] shape their religion through ritual practices tied to the sacred landscape » (p. ix). Peu après, il est question d'un tremblement de terre, en 1949, obligeant des gens à se réfugier dans une «montagne sacrée » (pp. 1-2). 


\section{AUTEURS}

\section{JUAN JAVIER RIVERA ANDÍA}

Université de Bonn 